\title{
MicroRNA-1908 is upregulated in human osteosarcoma and regulates cell proliferation and migration by repressing PTEN expression
}

\author{
HONGMOU YUAN and YANJUN GAO \\ Department of Orthopaedics, The Fourth Affiliated Hospital of China Medical \\ University, Shenyang, Liaoning 110032, P.R. China \\ Received April 13, 2015; Accepted July 27, 2015
}

DOI: $10.3892 /$ or.2015.4242

\begin{abstract}
Osteosarcoma is a high-grade malignant bone neoplasm. Although the introduction of chemotherapy has reduced its mortality, $>50 \%$ of patients develop chemoresistance and have an extremely poor prognosis due to pulmonary metastasis. Several molecular pathways contributing to osteosarcoma development and progression have recently been identified. Various studies have addressed the genes involved in the metastasis of osteosarcoma. However, the highly complex molecular mechanisms of metastasis remain to be elucidated. Recent studies have emphasized causative links between aberrant microRNA expression patterns and osteosarcoma progression. miR-1908 is dysregulated in certain human types of cancer. The expression pattern, clinical significance and biological role of miR-1908 in osteosarcoma, however, remain largely undefined. In the present study, we showed that miR-1908 was markedly upregulated in osteosarcoma cells and tissues compared with normal bone tissues using RT-qPCR. miR-1908 upregulation in osteosarcoma tissues was significantly associated with cell proliferation, invasion, advanced TNM stage and tumor growth. Both gainand loss-of-function studies showed that miR-1908 markedly increased the ability of osteosarcoma cells to proliferate and to invade through Matrigel in vitro. Analyses using mouse xenograft model revealed that xenografts of miR-1908 stableexpressing osteosarcoma cells exhibited a significant increase in tumor volume and weight, compared with the control group. Subsequent investigations revealed that miR-1908 directly inhibited the expression of phosphatase and tensin homolog deleted on chromosome ten (PTEN). Using a lucif-
\end{abstract}

Correspondence to: Hongmou Yuan, Department of Orthopaedics, The Fourth Affiliated Hospital of China Medical University, 4 Chongshan E Rd, Shenyang, Liaoning 110032, P.R. China

E-mail: yuanhongmou_cmu@163.com

Key words: miR-1908, osteosarcoma, invasion, PTEN erase reporter carrying the 3'-untranslated region (3'-UTR) of PTEN, we identified PTEN as a direct target of miR-1908. Collectively, the results showed that, miR-1908 promotes proliferation and invasion of osteosarcoma cells by repressing PTEN expression.

\section{Introduction}

Osteosarcoma is the most frequent primary malignant bone tumor in children and young adults (1). Although combined neoadjuvant chemotherapy has prolonged the survival rate greatly, the outcome of osteosarcoma patients with a poor response to chemotherapy is not encouraging. New strategies for treating recurrent and metastatic osteosarcoma remains a critical aim in clinical demands.

The focus of previous studies has been on the impact of microRNAs (miRNA, miR) on tumorigenesis and cancer progression (2-6). miRs are a class of small non-coding, single-stranded endogenous RNA fragments of 19-25 nucleotides (nt) in length that repress translation and cleaves mRNA by base-pairing to the untranslated region of the target gene. In various types of cancer, miRNA expression is significantly altered and this has potential to be a prominent diagnostic and prognostic tool (7). Elucidating the function of miRNAs in tumor pathogenesis and progression is important as they may play critical roles in the regulation of genes involved in controlling the development, proliferation/differentiation, apoptosis and drug resistance of tumor cells. Several studies $(2,8,9)$ have found that miR-1908 is dysregulated in certain types of human cancer. The expression pattern, clinical significance and biological role of miR-1908 in osteosarcoma, however, remains largely undefined.

In the present study, we showed that miR-1908 was markedly upregulated in osteosarcoma cells and tissues compared with normal bone tissues using RT-qPCR. miR-1908 upregulation in osteosarcoma tissues was significantly associated with cell proliferation, invasion, advanced TNM stage and tumor growth. Both gain- and loss-of-function studies showed that miR-1908 markedly increased the ability of osteosarcoma cells to proliferate and to invade through Matrigel in vitro. miR-1908 significantly increased the tumor volume and weight in vivo. Subsequent investigations revealed that miR-1908 directly 
inhibited the expression of phosphatase and tensin homolog deleted on chromosome ten (PTEN). Collectively, miR-1908 promotes the proliferation and invasion of osteosarcoma cells by repressing PTEN expression.

\section{Materials and methods}

Clinical specimens and cell culture. A total of 46 paraffinembedded osteosarcoma specimens and 9 normal muscle samples were obtained from the Fourth Affiliated Hospital of China Medical University and were used in accordance with the policies of the institutional review board of the hospital. All the diagnoses were confirmed by light microscopy and immunohistochemistry. No patient had received any antitumor treatments before biopsy. The human osteosarcoma cell lines (143B, U-2 OS, MG-63 and Saos-2) and hFOB 1.19 cell line were cultured in RPMI-1640 medium (Invitrogen Life Technologies, Carlsbad, CA, USA) supplemented with 10\% FBS (Gibco Life Technologies, Carlsbad, CA, USA) and $1 \%$ streptomycin/penicillin at $37^{\circ} \mathrm{C}$ with $5 \% \mathrm{CO}_{2}$.

RNA extraction, $R T-P C R$ and $R T-q P C R$. Total RNA was extracted from paraffin-embedded samples using acid phenol-chloroform method and from freshly-frozen samples or cells with TRIzol reagent (Invitrogen Life Technologies). Total RNA was reverse-transcribed using a First Strand cDNA Synthesis kit (Invitrogen Life Technologies). qPCR reactions were conducted using Platinum SYBR-Green qPCR SuperMix-UDG reagents (Invitrogen Life Technologies) on the Prism 7900HT system (Applied Biosystems, Foster City, CA, USA). Reactions were performed in triplicate and reactions without reverse transcriptase were used as negative controls. The U6 or GAPDH were used as the endogenous controls and the $2^{-\Delta \Delta \mathrm{CT}}$ equation was used to calculate the relative expression levels.

Oligonucleotide transfection and generation of stably transfected cell lines. Cells were seeded in 6-well plates, transfected with miR-1908 mimics or miR controls $(50 \mathrm{nM}$; GenePharma, Shanghai, China) using Lipofactamine ${ }^{\mathrm{TM}}$ RNAiMAX (Invitrogen Life Technologies). The cells were transfected with siMIF (100 nM; Invitrogen Life Technologies) or siRNA controls using Lipofactamine 2000 reagent (Invitrogen Life Technologies) and then harvested for assays $48 \mathrm{~h}$ later. The lentiviral plasmid pEZXMR03 (GeneCopoeia, Rockville, MD, USA) expressing 1908 (Cat, HmiR0274-MR03) or scrambled miRNA (Cat, CmiR0001-MR03) and Lenti-Pac HIV Expression Packaging mix (GeneCopoeia) were co-transfected into glioma cells using the EndoFectin Lenti transfection reagent (GeneCopoeia, Rockville, MD, USA). After transfection for $48 \mathrm{~h}$, lentiviral particles were harvested and then transduced into the glioma cells and the stably transfected cells were selected using puromycin and validated by RT-qPCR and western blot analysis.

MTT and colony formation assays. Osteosarcoma cells were seeded at a density of $3 \times 10^{3}$ cells/well in 96-well plates after transfection. An MTT assay was performed to test cell viability at 1,2, 3 and 4 days, and the absorbance was measured at
$490 \mathrm{~nm}$ with a spectrophotometric plate reader. For colony formation assay, the cells were plated at 500 cells/well in 6 -well plates after transfection and cultured for 14 days. Colonies were fixed with methanol, stained with $0.5 \%$ crystal violet and counted under the inverted microscope.

Western blot analysis. Equal amounts of protein were separated using SDS polyacrylamide gels and were then electrotransferred to polyvinylidene fluoride membranes (Millipore, Bedford, MA, USA). The membranes were immunoblotted overnight at $4^{\circ} \mathrm{C}$ with primary antibodies, followed by their respective secondary antibodies. $\beta$-actin was used as the loading control.

Cell migration and invasion assays. The effects of miR-1908 expression on cell migration and invasion were assessed using the wound-healing and Transwell assays as previously described (?).

In vivo tumor growth model. Male BALB/c athymic nude mice, aged 4-6 weeks, were purchased from the Hunan Slac Jingda Laboratory Animal Co., Ltd. (Changsha, China). For the tumor growth assay, cells stably overexpressing miR-1908 or scrambled miRNA were resuspended in PBS and $1 \times 10^{6}$ cells $(200 \mu 1)$ were subcutaneously injected in the dorsal flank of nude mice. Tumor size was measured every 3 days and tumor volumes were calculated using the formula: volume $=\left(\mathrm{LxW}^{2}\right) / 2$, in which $\mathrm{L}$ meant the longest diameter and $\mathrm{W}$ meant the shortest diameter. After 22 days, the mice were sacrificed and tumors were dissected and weighted. Animal handling and research protocols were approved by the Animal Care and Use Ethics Committee.

Immunohistochemical analysis. After 22 days, the mice were anesthetized and sacrificed, and tumors were removed, photographed, weighed and sectioned ( $5 \mathrm{~mm}$ in thickness), followed by immunostaining. Following deparaffinization, the sections were immunohistochemically analyzed using different antibodies, respectively, and subsequently were pathologically confirmed for the tumor phenotype and specific immunostaining. The positive cells were counted and analyzed.

Luciferase reporter assay. The 3'-UTR (untranslated region) sequence of PTEN was predicted to interact with miR-1908 or a mutated sequence within the predicted target sites was produced and inserted into the XbaI and FseI sites of the pGL3 control luciferase reporter vector (Promega, Madison, WI, USA). The luciferase reporter assay was performed as previously described (?).

Statistical analysis. Experimental data are shown as mean \pm standard deviation (SD). The results from different treatment groups were compared using a two-tailed Student's t-test. The Kaplan-Meier method was used to estimate the probability of the patient survival rate. A correlation analysis was performed to determine the association between miR-1908 and the patients survival rate. Differences were considered to be statistically significant at $\mathrm{P}<0.05$. Statistical analysis was performed with SPSS/Win11.0 software (SPSS, Inc., Chicago, Illinois, USA). 

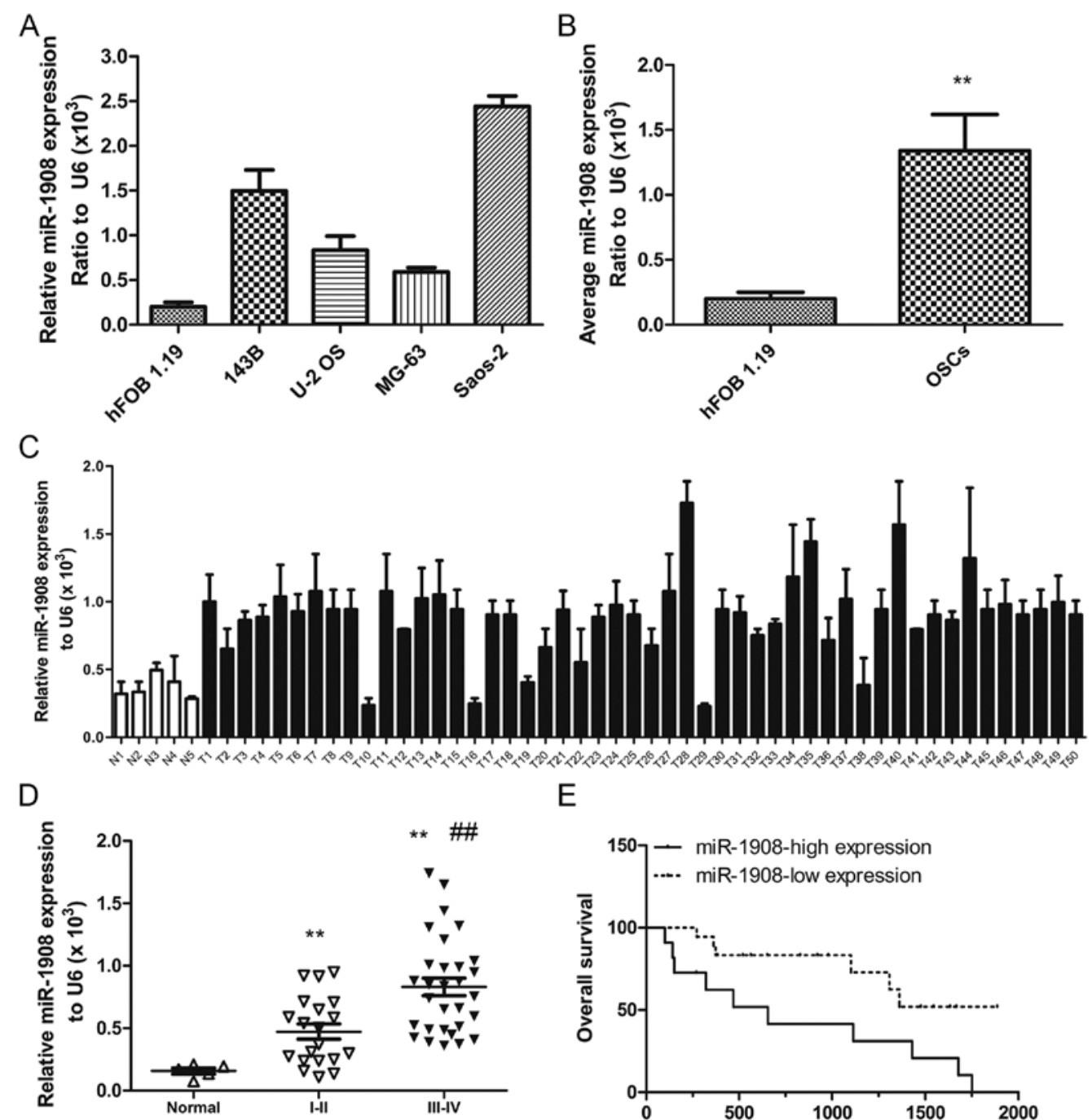

$\mathrm{E}$

Figure 1. Aberrant expression of miR-1908 in human osteosarcoma cells and tumors. (A) Quantification of miR-1908 in osteosarcoma cells lines shows a higher level than that in normal osteoblast cell line. (B) Average expression level of miR-1908 in osteosarcoma cells lines. (C) Quantification of miR-1908 in tumor specimens and normal muscle tissues. (D) Quantification of miR-1908 in normal muscle tissues, osteosarcoma of stages I-II and III-IV. (E) Correlation analysis of miR-1908 and overall patient survival rate shows that miR-1908 level is a risk indicator for survival. ${ }^{* *} \mathrm{P}<0.01$ and ${ }^{\# \#} \mathrm{P}<0.01$.

\section{Results}

Aberrant expression of miR-1908 in human osteosarcoma cells and tumors. In order to confirm whether miR-1908 is associated with osteosarcoma, miR-1908 levels were measured in osteosarcoma cell lines (143B, U-2 OS, MG-63 and Saos-2) and a normal osteoblast cell line (hFOB 1.19), as well as in human tumor specimens $(\mathrm{n}=46)$ and normal muscle tissues $(\mathrm{n}=9)$. The results showed that, miR-1908 was significantly higher in osteosarcoma cells (Fig. 1A and B) than in the normal osteoblast cell line and significantly higher in osteosarcoma tumors than in the normal muscle tissue (Fig. 1C). miR-1908 was highest in stage III-IV tumors and higher in stage I-II tumors as compared to normal muscle tissue (Fig. 1D). miR-1908 was also associated with the overall patient survival rate. Furthermore, more patients with lower miR-1908 survived following treatment than those with higher miR-1908 (Fig. 1E). Collectively, these data demonstrated that miR-1908 is upregulated in osteosarcoma and that higher miR-1908 expression predicts a poor overall patient survival rate.
Overexpressed miR-1908 promotes osteosarcoma cell proliferation, invasion and sphere formation. MTT and colony formation assays were carried out to assess the functional role of miR-1908 in osteosarcoma cells by determining the effects of miR-1908 overexpression in MG-63 and U-2 OS cell lines. Overexpression of miR-1908 in MG-63 and U-2 OS resulted in a higher growth rate as compared with the controls (Fig. 2A). In addition, the colony formation assay revealed that miR-1908 promoted the viability of osteosarcoma cells as compared with the controls (Fig. 2B). Collectively, these results suggested that miR-1908 is an important regulator of proliferation in osteosarcoma cells. The effect of miR-1908 on cell migration was first assessed by the wound-healing assay. MG-63-miR-1908 and U-2 OS-miR-1908 cells had a significantly faster closure of the wound area compared to their control cells (Fig. 2C). This result was confirmed by Boyden's chamber assay (Fig. 2D). Moreover, MG-63-miR-1908 and U-2 OS-miR-1908 cells showed a greater degree of invasion through Matrigel (Fig. 2D). We then analyzed the effects of miR-1908 on osteosarcoma self-renewal using a sphere 
A

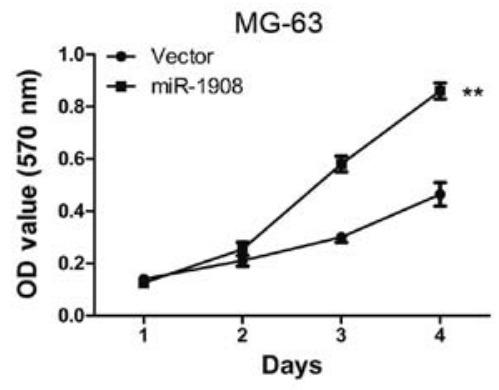

B

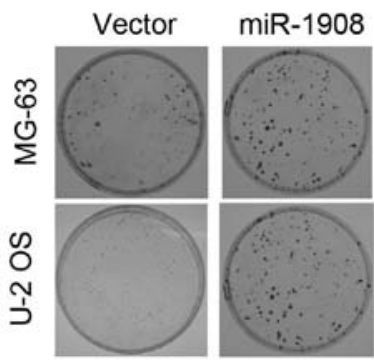

$\mathrm{U}-2$ OS
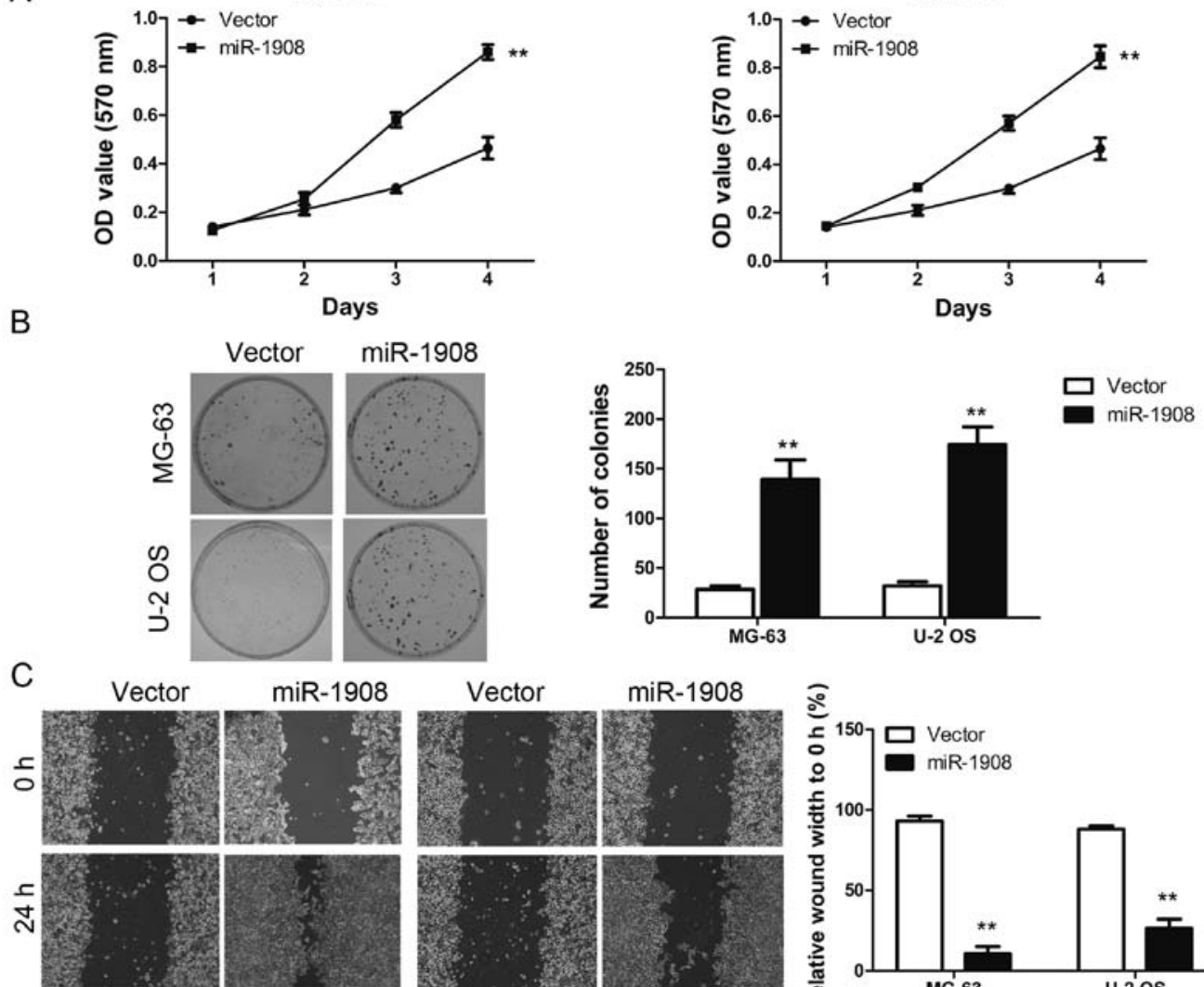

MG-63

D

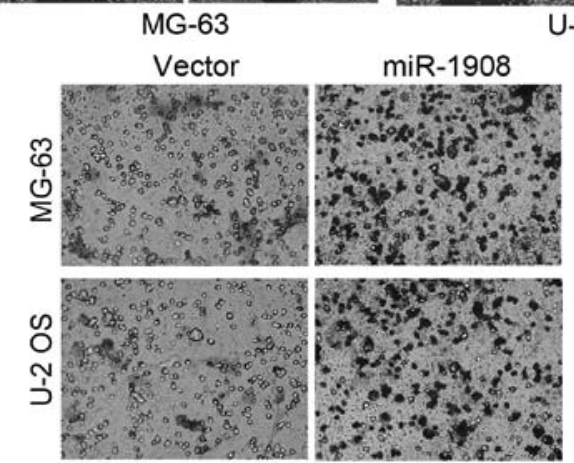

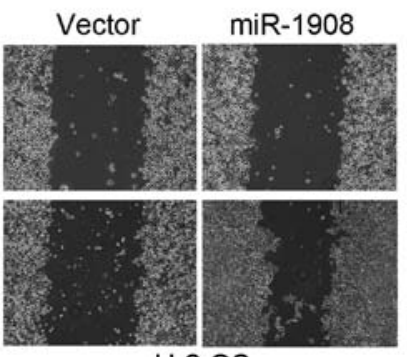

$\mathrm{U}-2 \mathrm{OS}$
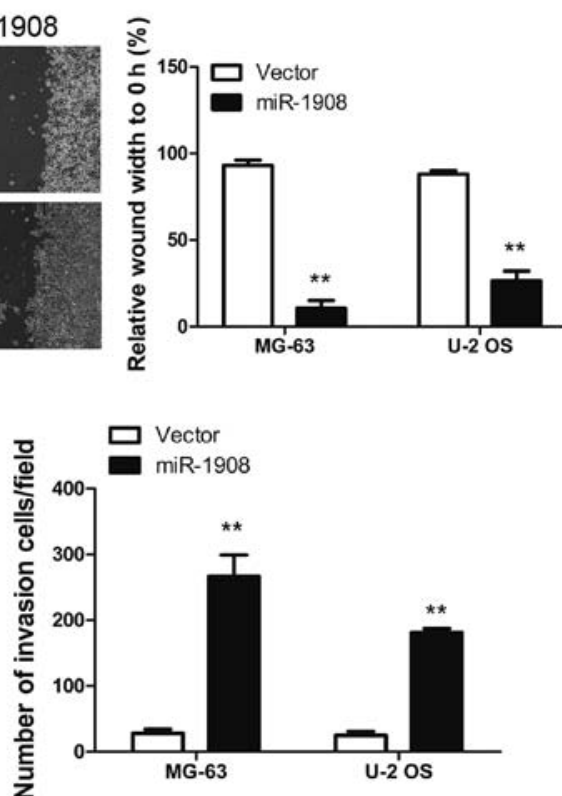

Figure 2. Overexpressed miR-1908 promotes osteosarcoma cell proliferation and invasion. (A) MTT assay shows higher cell proliferation rates in MG-63 and U-2 OS of miR-1908 overexpression. (B) Colonies were stained with crystal violet in the colony formation assay for 14 days. (C) Wound-healing assay results show that MG-63-miR-1908 and U-2 OS-miR-1908 cells had significantly faster closure of the wound area. (D) Boyden's chamber assay results show a greater degree of invasion through Matrigel in MG-63-miR-1908 and U-2 OS-miR-1908 cells. ${ }^{* *} \mathrm{P}<0.01$.

A
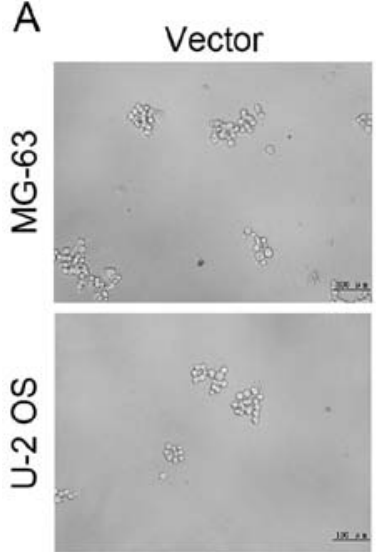

miR-1908

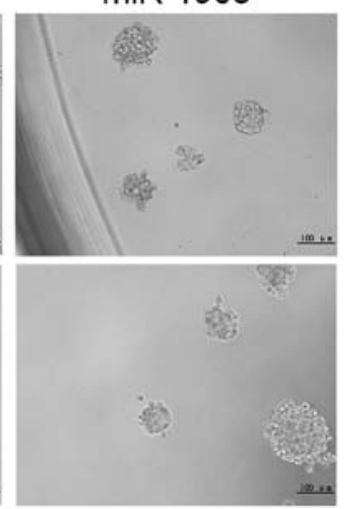

B

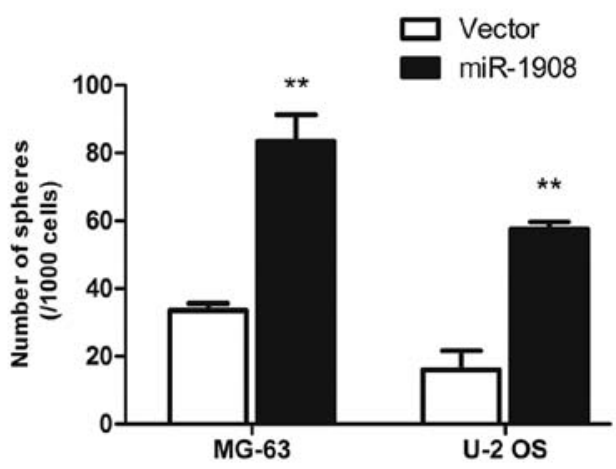

Figure 3. Overexpressed miR-1908 promotes osteosarcoma cell sphere formation. (A) Sphere formation after one week results in increased sphere size and number in MG-63-miR-1908 and U-2 OS-miR-1908 cells. (B) Analysis of vector groups and miR-1908 overexpression groups ("* $\mathrm{P}<0.01)$. 
A

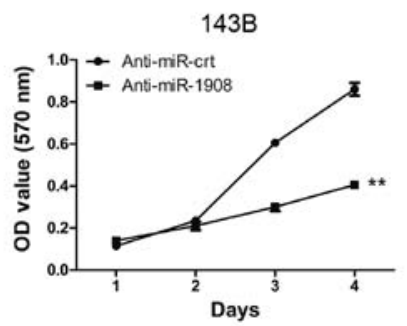

Saos-2

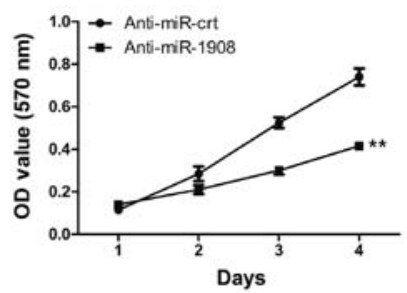

B

B Anti-miR-crt Anti-miR-1908

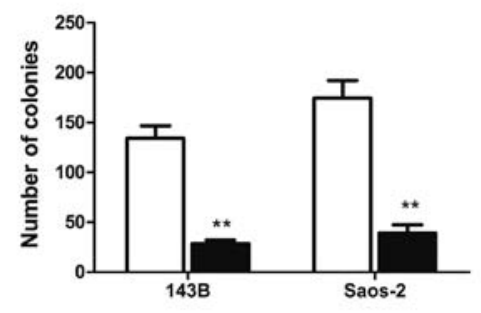

C
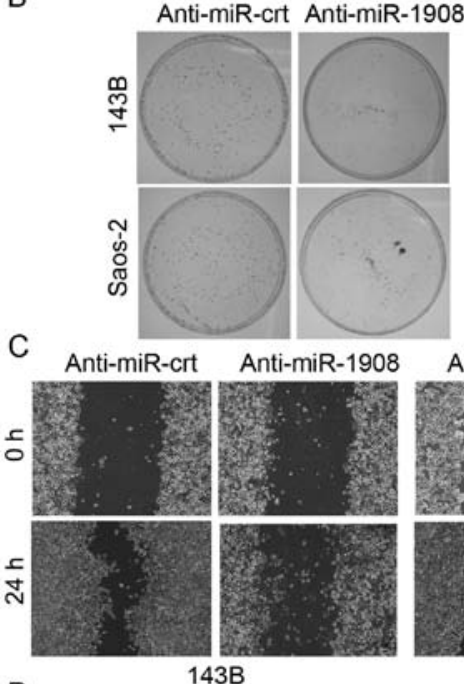

D
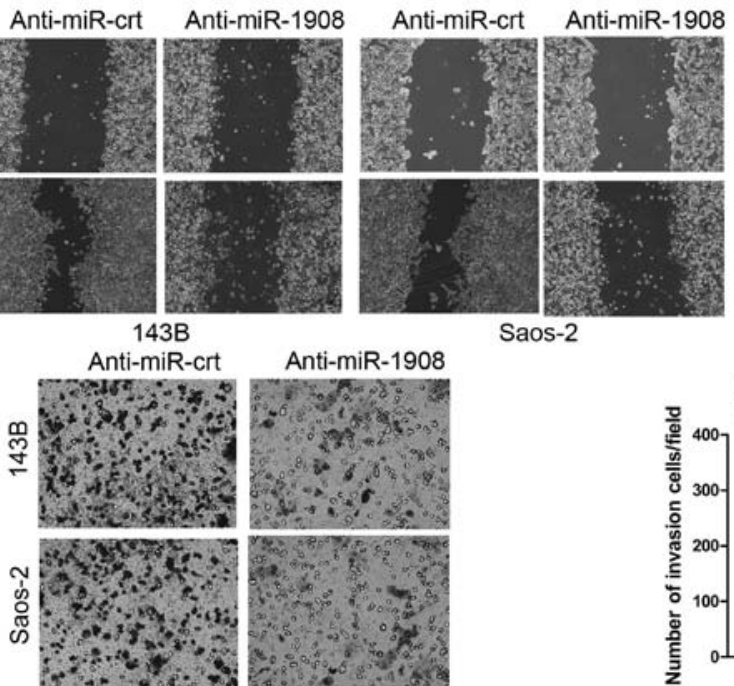

Saos-2

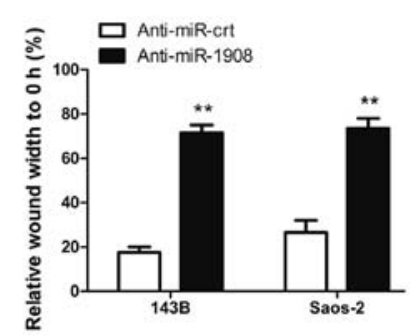

$\square$ Anti-miR-crt

Anti-miR-1908

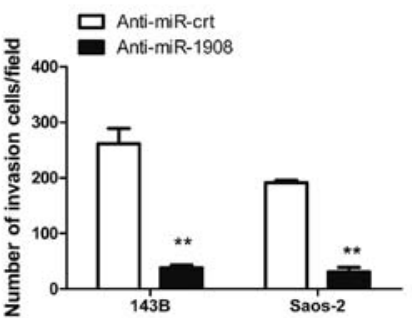

Figure 4. Silencing of miR-1908 inhibits osteosarcoma cell proliferation and invasions. (A) MTT assay shows lower cell proliferation rates in the 143B-Anti-miR-1908 and Saos-2-Anti-miR-1908 groups. (B) Colonies were stained with crystal violet in the colony formation assay for 14 days. (C) Wound-healing assay results show that 143B-Anti-miR-1908 and Saos-2-Anti-miR-1908 cells had a significantly slower closure of the wound area. (D) Boyden's chamber assay results show a lower degree of invasion through Matrigel in 143B-Anti-miR-1908 and Saos-2-Anti-miR-1908 cells. ${ }^{* *} \mathrm{P}<0.01$.

formation assay. miR-1908 was transfected into osteosarcoma cells and sphere formation was assessed for one week. miR-1908 overexpression increased the sphere size and number (Fig. 3A and B). These data suggested that miR-1908 promotes the self-renewal ability of osteosarcoma cells.

Silencing of miR-1908 inhibits osteosarcoma cell proliferation, invasion and sphere formation. We determined whether the silencing of miR-1908 inhibits the malignant phenotype of osteosarcoma cells. Silencing of miR-1908 in $143 \mathrm{~B}$ and Saos-2 cells significantly reduced cell proliferation (Fig. 4A) and clonogenicity (Fig. 4B). The effect of silencing of miR-1908 on cell migration was first assessed by a wound-healing assay.

The 143B-Anti-miR-1908 and Saos-2-Anti-miR-1908 cells reduced the migratory capacity compared to their control cells (Fig. 4C). This result was confirmed by the Boyden's chamber assay (Fig. 4D). In addition, 143B-Anti-miR-1908 and Saos-2-Anti-miR-1908 cells showed a lower degree of invasion through Matrigel (Fig. 4D). The sphere formation assay showed that silencing of miR-1908 decreased sphere size and number (Fig. 5A and B). This result indicated that miR-1908 promotes the self-renewal ability of osteosarcoma cells. Collectively, the results suggested that miR-1908 is an important regulator of proliferation in osteosarcoma cells.

Silencing of miR-1908 inhibits osteosarcoma tumor formation in vivo. To confirm whether the biologic effect of miR-1908 observed in cultured cells is relevant to osteosarcoma growth in vivo, 143B-Anti-miR-1908 and control, respectively, were subcutaneously inoculated into BALB/C athymic nude mice. As shown in Fig. 6A, tumors formed by miR-1908-silencing cells grew more slowly than those by the vector control cells following inoculation and the difference in average tumor volume between experimental and control animals continued to increase 2 -fold at the experimental endpoint (22 days) (Fig. 6A and B). Concurrently, increases in sizes and weights of tumors excised from animals of the miR-1908 overexpression group were also observed as compared with those of the control group (Fig. 6B and C). Consistent with the above 
A

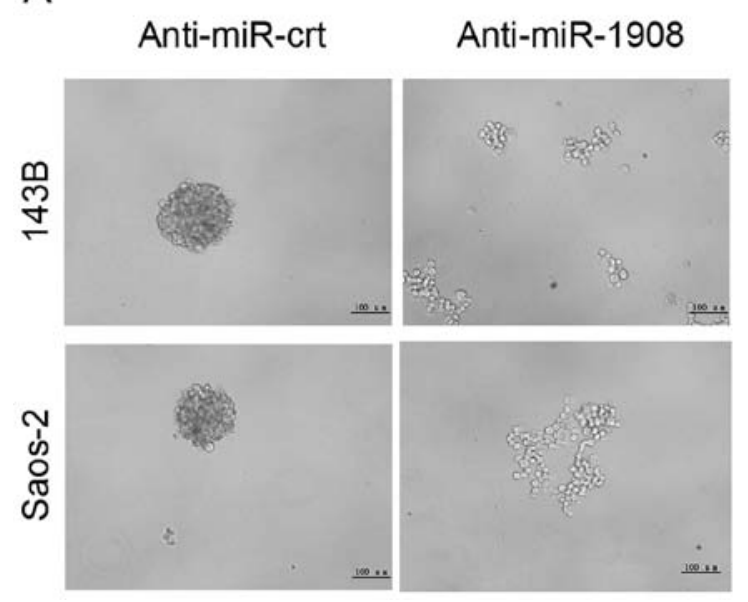

B

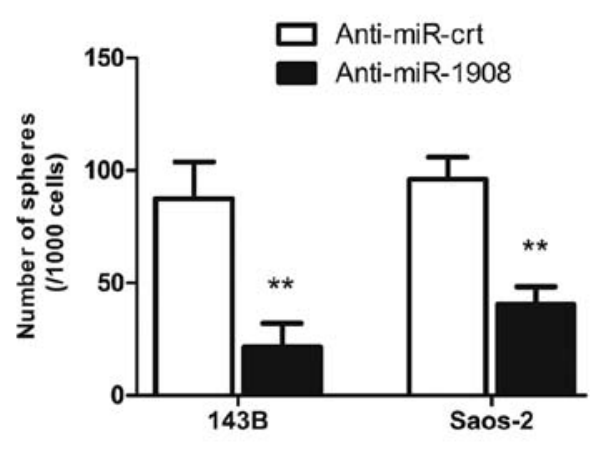

Figure 5. Silencing of miR-1908 inhibits osteosarcoma cell sphere formation. (A) Sphere formation after one week results in reduced sphere size and number in 143B-Anti-miR-1908 and Saos-2-Anti-miR-1908 cells. (B) Analysis of control and miR-1908 knockdown groups ( $\left(^{* *} \mathrm{P}<0.01\right)$.

A

C
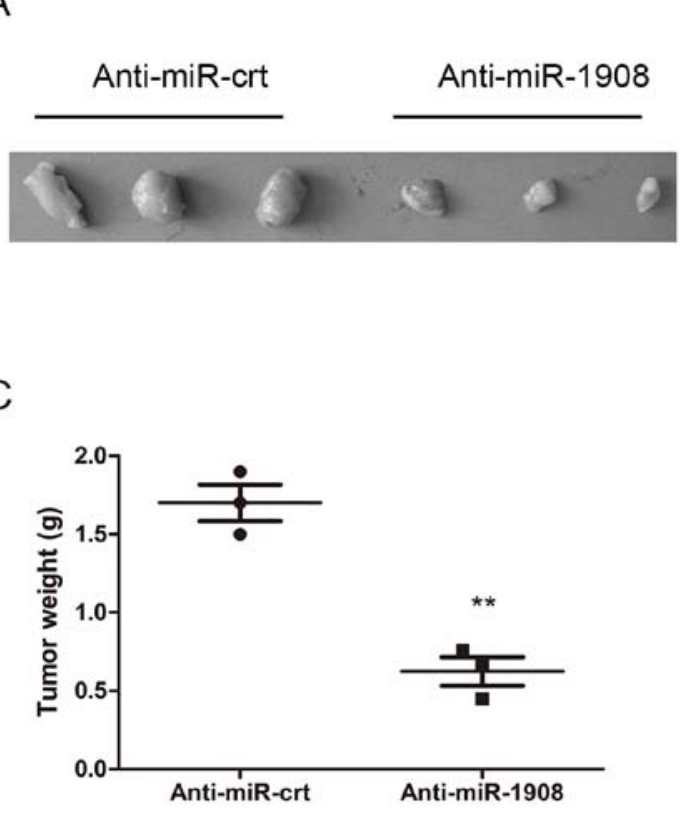

$\mathrm{E}$

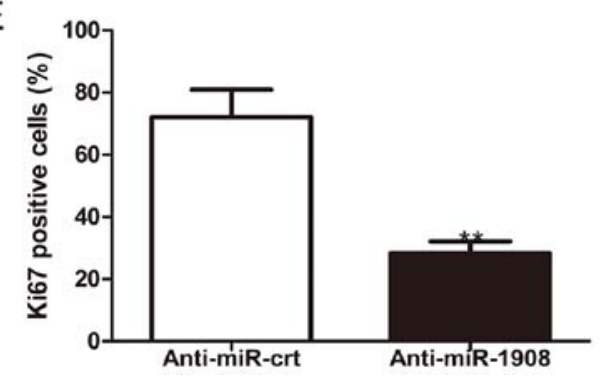

B $1000 \rightarrow-$ Anti-miR-crt

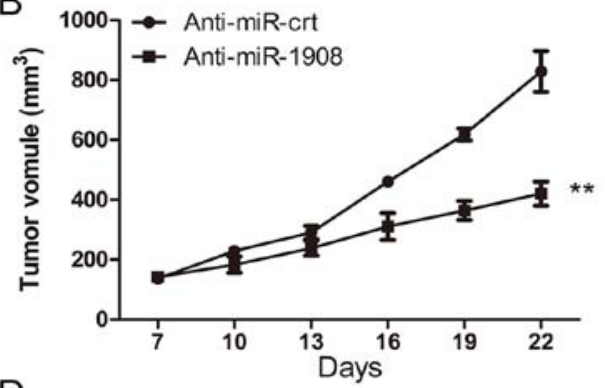

D $\quad \mathrm{HE}$

Ki67

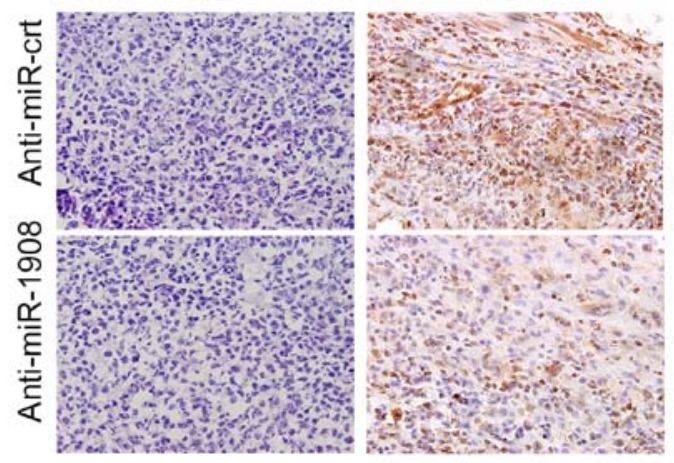

Figure 6. Silencing of miR-1908 inhibits osteosarcoma tumor formation in vivo. (A) Tumor formed are evident. Tumors in the miR-1908 knockdown group are smaller than those of the control group. (B) The growth curves of tumor volumes. (C) Analysis of tumor weights. (D) HE staining and immunohistochemical staining of Ki67. (E) Analysis of Ki67-positive cells in the control and miR-1908 knockdown groups. ${ }^{* *} \mathrm{P}<0.01$.

observations, as shown in Fig. 6D and E, the proportion of Ki67-positive cells increased in the control tumors compared to that in miR-1908-silenced tumors.
miR-1908 directly targets PTEN and PTEN levels are inversely correlated with miR-1908 levels in osteosarcoma tissues. To reveal mRNA targets of miR-1908 in osteosarcoma, we used 
A

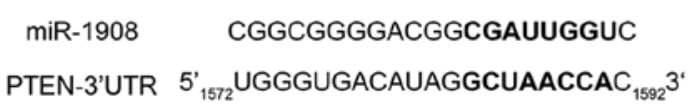

C

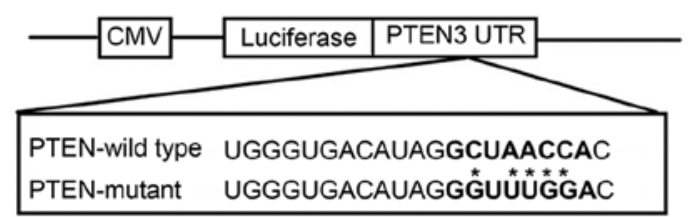

D

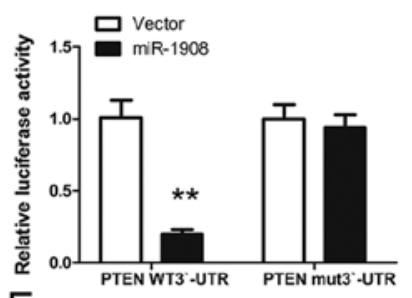

F

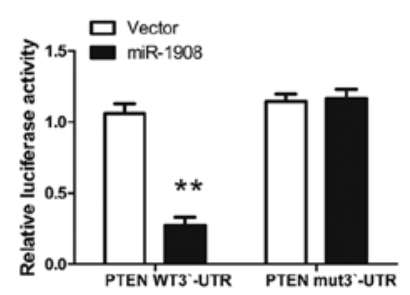

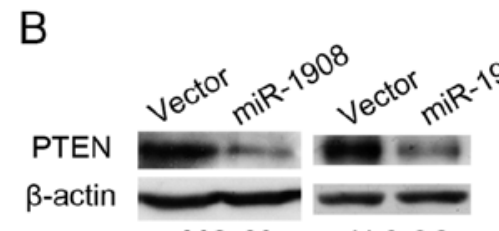

MG-63

U-2 OS

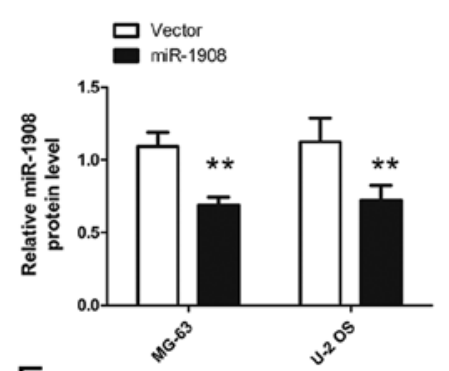

$\mathrm{E}$

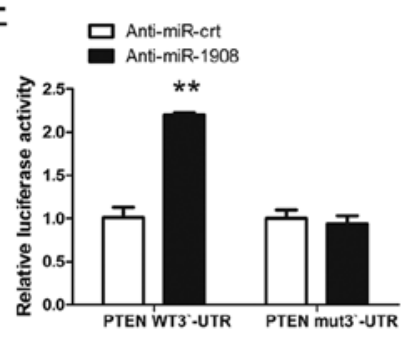

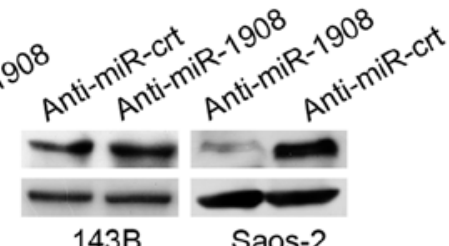

Saos-2
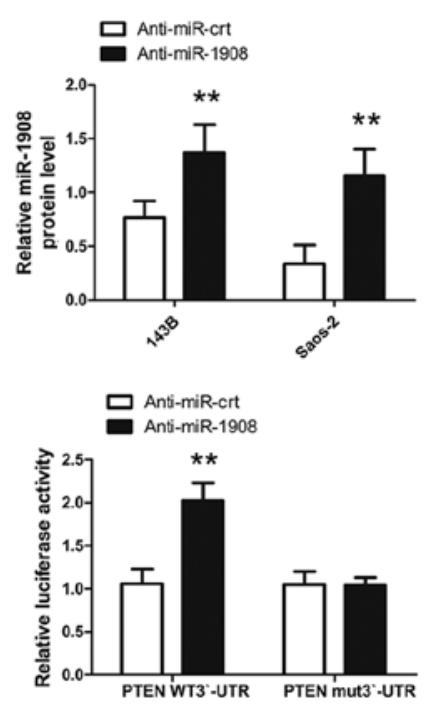

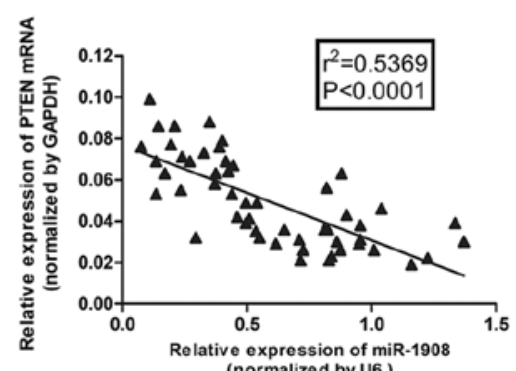

(normalized by U6)

Figure 7. miR-1908 directly targets PTEN and PTEN levels are inversely correlated with miR-1908 levels in osteosarcoma tissues. (A) Sequences of miR-1908 and PTEN 3'-UTR. (B) PTEN protein level in each group and corresponding analysis. (C) Sequences of wild-type and mutant-type of PTEN 3'-UTR. (D and E) Relative luciferase activity of PTEN in cells after co-transfection with wild-type (WT) or mutant (mut) PTEN 3'-UTR reporter genes and miR-1908 mimics, anti-miR-1908 mimics or control. (F) Correlation analysis of miR-1908 overexpression with PTEN downregulation. ${ }^{* *} \mathrm{P}<0.01$

bioinformatics databases (TargetScan, Pictar, RNAhybrid) to identify potential tumor-suppressor targets. The PTEN (NM_000314) gene contained the predicted binding site for miR-1908 (Fig. 7A). To experimentally verify this potential target, the cells were transfected with miR-1908 and the protein and mRNA target levels were assessed by western blot analysis. miR-1908 overexpression reduced and miR-1908 knockdown increased the expression of PTEN in osteosarcoma cells (Fig. 7B). To determine whether PTEN 3'-UTR is a direct target of miR-1908, PTEN 3'-UTR reporter constructs or 3'-UTR mutant controls were transfected into osteosarcoma cells before transfection with miR-1908 and luciferase activity was measured (Fig. 7C). As shown in Fig. 7D and E, the luciferase activity was significantly decreased in osteosarcoma cells overexpressing miR-1908 co-transfected with 3'-UTR-PTEN-wt vector and miR-1908 mimic compared with those co-transfected with 3'-UTR-PTEN-mut vector and
miR-1908 mimic. By contrast, increased activity was observed in osteosarcoma cells silencing miR-1908, suggesting that the fragment at the 3'-UTR of the PTEN was the complementary site for the miR-1908 seed region, and thus, that PTEN was a direct target of miR-1908. In addition, PTEN expression was inversely proportional to miR-1908 expression (Fig. 7F). Collectively, miR-1908 directly affected PTEN expression.

\section{Discussion}

Osteosarcoma is one of the most common malignancies and one of the leading causes of cancer-related mortality worldwide $(10,11)$. Despite recent advances in disease management and treatment, osteosarcoma patients have a poor long-term prognosis. The main challenges in the treatment of osteosarcoma involve recurrence and metastasis, leading to the prediction poor outcome for osteosarcoma patients. 
The identification of critical molecules that suppress these processes may lead to novel therapeutic targets for improving the prognosis of these patients. Various studies have identified some key signaling transduction cascades that are involved in the progression, invasion and metastasis of osteosarcoma, such as p53 $(12,13)$, the Ras/MAPK pathways $(14,15)$ and the PTEN pathway (16-18). However, the underlying molecular mechanisms of osteosarcoma metastasis remain to be determined.

PTEN is located at 10q23.3 and encodes a dual-specificity phosphatase with lipid and protein phosphatase activities. PTEN suppressed the migration and genetic deletion of the PTEN tumor suppressor gene, promoting cell motility and overexpression or reconstitution of PTEN and inhibiting cell motility in a variety of cell types (19-21). Mechanistically, PTEN repressed cell motility through a variety of pathways and PI3K/AKT is an important target of PTEN. miRNAs have been shown to play an important role in the maintenance of normal cell function and the dysregulation of miRNA expression may result in cancer initiation and tumor progression. Hundreds of genes were found to be the target of miRNA, which harbors the target sequence in their 3'-UTR complementary to the seed region of the miRNA. Previous studies have reported that certain miRNAs can directly target PTEN (22-24).

miR-1908 is a new member of the microRNA family. Although results of a previous study indicate that miR-1908 promoted invasion and angiogenesis in melanoma (25), angiogenesis has not been investigated in this study. In this study, the expression, function, and mechanisms of action of miR-1908 in osteosarcoma were investigated. We found that miR-1908 was a risk factor in osteosarcoma where it acts as an oncogene by regulating PTEN expression. In the present study, it was demonstrated that miRNA-1908 targets PTEN, resulting in the reduced expression of PTEN and an increase in migration and proliferation of osteosarcoma cells in vitro. In osteosarcoma cells, miR-1908 overexpression is associated with higher cell proliferation and invasion in vitro. By contrast, silencing endogenous miR-1908 markedly abrogated the proliferation and invasion of osteosarcoma cells. miR-1908 overexpression and knockdown cell lines were established separately. miR-1908 overexpression is associated with higher cell proliferation and migration, while silencing of miR-1908 is associated with lower cell proliferation and migration. Of note, the close correlation between a high miR-1908 expression and low expression of PTEN, as well as with the malignant properties of osteosarcoma, was also observed in athymic nude mice and in clinical osteosarcoma samples, suggesting a possible role of miR-1908 in the development and progression of osteosarcoma. miR-1908 directly targeted PTEN and PTEN levels are inversely correlated with miR-1908 levels in osteosarcoma tissues.

In summary, results of the present study have demonstrated that miR-1908 is overexpressed in osteosarcoma cells in vitro. Concurrently, miR-1908 overexpression is associated with poor patient survival. The present study presents evidence that indicates that miR-1908 has oncogenic, proliferation, migration and invasion regulatory effects that are mediated by PTEN in osteosarcoma cells. The present study therefore provides, to the best of our knowledge, a first characterization of miR-1908 as an oncogene and a potential therapeutic target in osteosarcoma.

\section{Acknowledgements}

The present study was supported by the Science and Technology Research Foundation of Education Department of Liaoning Province (no. L2010661) and the Science and Technology Projects of Technology Department of Liaoning province (no. 2013021015).

\section{References}

1. Mirabello L, Troisi RJ and Savage SA: Osteosarcoma incidence and survival rates from 1973 to 2004: Data from the Surveillance, Epidemiology, and End Results Program. Cancer 115: 1531-1543, 2009.

2. Long C, Jiang L, Wei F, Ma C, Zhou H, Yang S, Liu X and Liu Z: Integrated miRNA-mRNA analysis revealing the potential roles of miRNAs in chordomas. PLoS One 8: e66676, 2013.

3. Farazi TA, Hoell JI, Morozov P and Tuschl T: MicroRNAs in human cancer. Adv Exp Med Biol 774: 1-20, 2013.

4. Hsu SH, Wang B, Kota J, Yu J, Costinean S, Kutay H, Yu L, Bai S, La Perle K, Chivukula RR, et al: Essential metabolic, anti-inflammatory, and anti-tumorigenic functions of miR-122 in liver. J Clin Invest 122: 2871-2883, 2012.

5. Fang Y, Xue JL, Shen Q, Chen J and Tian L: MicroRNA-7 inhibits tumor growth and metastasis by targeting the phosphoinositide 3-kinase/Akt pathway in hepatocellular carcinoma. Hepatology 55: 1852-1862, 2012.

6. Volinia S, Calin GA, Liu CG, Ambs S, Cimmino A, Petrocca F, Visone R, Iorio M, Roldo C, Ferracin M, et al: A microRNA expression signature of human solid tumors defines cancer gene targets. Proc Natl Acad Sci USA 103: 2257-2261, 2006.

7. Rawlings-Goss RA, Campbell MC and Tishkoff SA: Global population-specific variation in miRNA associated with cancer risk and clinical biomarkers. BMC Med Genomics 7: 53, 2014.

8. Pencheva N, Tran H, Buss C, Huh D, Drobnjak M, Busam K and Tavazoie SF: Convergent multi-miRNA targeting of ApoE drives LRP1/LRP8-dependent melanoma metastasis and angiogenesis. Cell 151: 1068-1082, 2012.

9. Jin JC, Jin XL, Zhang X, Piao YS and Liu SP: Effect of OSW-1 on microRNA expression profiles of hepatoma cells and functions of novel microRNAs. Mol Med Rep 7: 1831-1837, 2013.

10. Geller DS and Gorlick R: Osteosarcoma: A review of diagnosis, management, and treatment strategies. Clin Adv Hematol Oncol 8: 705-718, 2010.

11. Zhang Y, Zhang L, Zhang G, Li S, Duan J, Cheng J, Ding $\mathrm{G}$, Zhou C, Zhang J, Luo P, et al: Osteosarcoma metastasis: Prospective role of ezrin. Tumour Biol 35: 5055-5059, 2014.

12. He Y, de Castro LF, Shin MH, Dubois W, Yang HH, Jiang S, Mishra PJ, Ren L, Gou H, Lal A, et al: p53 loss increases the osteogenic differentiation of bone marrow stromal cells. Stem Cells 33: 1304-1319, 2015.

13. Diller L, Kassel J, Nelson CE, Gryka MA, Litwak G, Gebhardt M, Bressac B, Ozturk M, Baker SJ, Vogelstein B, et al: p53 functions as a cell cycle control protein in osteosarcomas. Mol Cell Biol 10: 5772-5781, 1990.

14. Medema RH, Kops GJ, Bos JL and Burgering BM: AFX-like Forkhead transcription factors mediate cell-cycle regulation by Ras and PKB through p27kip1. Nature 404: 782-787, 2000.

15. Zheng Z, Ding M, Ni J, Song D, Huang J and Wang J: MiR-142 acts as a tumor suppressor in osteosarcoma cell lines by targeting Rac1. Oncol Rep 33: 1291-1299, 2015.

16. Hu Y, Xu S, Jin W, Yi Q and Wei W: Effect of the PTEN gene on adhesion, invasion and metastasis of osteosarcoma cells. Oncol Rep 32: 1741-1747, 2014.

17. Song D, Ni J, Xie H, Ding M and Wang J: DNA demethylation in the PTEN gene promoter induced by 5 -azacytidine activates PTEN expression in the MG-63 human osteosarcoma cell line. Exp Ther Med 7: 1071-1076, 2014.

18. Shen L, Chen XD and Zhang YH: MicroRNA-128 promotes proliferation in osteosarcoma cells by downregulating PTEN. Tumour Biol 35: 2069-2074, 2014. 
19. Yang YK,Xi WY,XiRX,Li JY,Li Q and Gao YE: MicroRNA-494 promotes cervical cancer proliferation through the regulation of PTEN. Oncol Rep 33: 2393-2401, 2015.

20. Zhang WL and Zhang JH: miR-181c promotes proliferation via suppressing PTEN expression in inflammatory breast cancer. Int J Oncol 46: 2011-2020, 2015.

21. Hodakoski C, Fine B, Hopkins B and Parsons R: Analysis of intracellular PTEN signaling and secretion. Methods 77-78: 164-171, 2015.

22. Zhang LY, Ho-Fun Lee V, Wong AM, Kwong DL, Zhu YH, Dong SS, Kong KL, Chen J, Tsao SW, Guan XY, et al: MicroRNA-144 promotes cell proliferation, migration and invasion in nasopharyngeal carcinoma through repression of PTEN. Carcinogenesis 34: 454-463, 2013.
23. Bar N and Dikstein R: miR-22 forms a regulatory loop in PTEN/AKT pathway and modulates signaling kinetics. PLoS One 5: e10859, 2010

24. Garofalo M, Di Leva G, Romano G, Nuovo G, Suh SS, Ngankeu A, Taccioli C, Pichiorri F, Alder H, Secchiero P, et al: miR-221\&222 regulate TRAIL resistance and enhance tumorigenicity through PTEN and TIMP3 downregulation. Cancer Cell 16: 498-509, 2009.

25. Pencheva N, Tran H, Buss C, et al: Convergent multi-miRNA targeting of ApoE drives LRP1/LRP8-dependent melanoma metastasis and angiogenesis. Cell 151: 1068-1082, 2012. 